In continuation of Stamm's work, we have deter. mined the molecular weights (by sedimentation equilibrium in the Svedberg ultracentrifuge), and we find that the specific viscosity increases for a number of celluloses and regenerated celluloses dissolved in cuprammonium solvent. Calculating the results in the same manner as Stamm did, we obtain apparent molecular weights of the cellulose-copper complex ranging from 100,000 to 300,000 , depending upon the extent to which degradation had occurred during the previous history of the specimens. The 300,000 value was obtained for a portion of cellulose which Stamm studied, and for which he reported a value of 55,000 for the cellulose-copper complex. We suspect that degradation inadvertently occurred during Stamm's determinations. To obtain the molecular weight on a copper-free basis, Stamm assumed one copper atom combined per glucose group, corresponding to a correction factor of 72 per cent and leading to his final value of 40,000 . Our results on combined copper, and also consideration of the partial specific volume of the copper compound, yield a correction factor of 60 per cent, so that our cellulose molecular weights range from 60,000 to 180,000 . We estimate the molecular weight of native cellulose to be in the neighbourhood of 300,000 .

The specific viscosity of the cuprammonium solutions of the celluloses increases in a definite manner with the molecular weight, so that after empirical calibration of the relationship by ultracentrifugal analysis, it is possible to calculate average molecular weights from viscosity data. The numerical relationship in the range of molecular weight that we have studied varies appreciably with the composition of the solvent and is not in agreement with the relationship published by Staudinger. The application of Staudinger's equation to our viscosity data gives molecular weight values of 20,000 to 90,000 , that is, from a third to a half as great as the ultracentrifuge values.

Elmer O. KraEmer.

William D. LaNSING.

Experimental Station,

E. I. du Pont de Nemours and Co.,

Wilmington, Delaware.

April 26.

\footnotetext{
1 H. Mark, Trans. Faraday Soc., 29, 41; 1933.

2 A. J. Stamm, J. Amer. Chem. Soc., 52, 3047; 1930.

3 H. Staudinger, Trans. Faraday Soc., 2
}

\section{Natural Interconversion of Isomeric Sugars}

THE mechanism involved in the smooth trans. formation of one simple sugar into another is a matter of the utmost importance to the chemist and the physiologist alike, but little light was thrown upon the subject until Robinson ${ }^{1}$ introduced the interesting theory that Walden inversion (conditioned by the enzymatic hydrolysis of phosphoric esters) within the sugar molecule is an agency for such changes. This hypothesis presents a simple and rational explanation of the conversion of glucose into galactose by the mammary glands during lactation, and lends colour to the suggestion that the primary constituent of nucleic acid is the commonly-occurring xylose, which undergoes conversion to ribose in an analogous manner. Mathers and Robertson $^{2}$, in a research on the hydrolysis of $p$-toluenesulphonyl esters of glucose, recently adduced evidence which strongly supported this view, in as much as they were able to convert a derivative of glucose into a derivative of altrose in one operation. Cognate researches have brought to light the following significant facts.

The alkaline hydrolysis of :

(1) $2: 3$-Di-p-toluenesulphonyl $4: 6$-dimethyl $\alpha$-methylglucoside yields a $2: 3$-anhydro $4: 6$ dimethyl $\alpha$-methylhexoside and $4: 6$-dimethyl $\alpha$-methylaltroside ${ }^{2}$.

(2) 2 : 3-Di-p-toluenesulphonyl $4: 6$-benzylidene $\alpha$-methylglucoside yields a $2: 3$-anhydro $4: 6$-benzylidene $\alpha$-methylhexoside and a monomethyl $4: 6$ benzylidine $\alpha$-methylhexoside which is not a derivative of glucose or mannose.

(3) $2: 3$-Dimethyl $4: 6$-di- $p$-toluenesulphonyl $\alpha$-methylglucoside yields a complicated mixture containing a derivative of glucoseen.

(4) 3-p.Toluenesulphonyl diacetone glucose gives a quantitative yield of diacetone glucose.

(5) 2 : 3-Dimethyl 4-p-toluenesulphonyl 6-triphenylmethyl $\alpha$-methylglucoside gives an almost quantitative yield of $2: 3$-dimethyl 6-triphenylmethyl $\alpha$-methylglucoside.

(6) $2: 3: 6$-Trimethyl 4-p-toluenesulphonyl $\beta$-methylglucoside gives unchanged material and $2: 3: 6$-trimethyl $\beta$-methylglucoside.

Viewing the results as a whole, it is evident that these hydrolytic reactions fall into two main groups, which may be characterised as normal and abnormal, according as the original substance contains one or two $p$-toluenesulphonyl residues in neighbouring positions. It is also worthy of note that in the cases where Walden inversion has been proved to occur, such inversion is accompanied by anhydro formation. This fact at once suggests the idea that anhydro formation may be a necessary precursor to this type of inversion, which follows as a consequence of the opening of the anhydro ring. Such a hypothesis is in keeping with the main principle of the Robinson conception, and at the same time invalidates the criticism levelled against it by Levene ${ }^{3}$, whose evidence is based upon the hydrolysis of a phosphoric ester of 5-methyl monacetone xylose, in which the possibility of anhydro formation is precluded.

A full account of these investigations will be published later, and it is hoped that the various extensions of the work which are now on hand will lead to a definite elucidation of this complicated but highly important problem.

G. J. Robertson.
J. W. H. Oldham.
Chemical Research Laboratory,
University of St. Andrews.
April 27.

1 Robinson, Nature, 120, 44, July 9, 1927.

Mathers and Robertson, J. Chem. Soc., 1076 ; 1933. cf. NATURE, 132,789 , Nov. $18,1933$.

${ }^{3}$ Levene and Raymond, J. Biol. Chem., 102, 347 ; 1933.

\section{A New Type of Artificial $\beta$-Radioactivity}

THE energy spectrum of positive electrons from magnesium, when bombarded by $\alpha$-particles of radium $C^{\prime}$ reduced in range to $6 \cdot 3 \mathrm{~cm}$., were investigated by a method already described ${ }^{1}$. It was found that the number of positives is less than that expected from the measurements of the integral effect. Changing the direction of the magnetic field, a great number of negative electrons could be observed. The number of negative electrons was about four times as great as the number of positive electrons. 